\title{
Sello de calidad profesional en atención temprana
}

\section{The professional quality seal for early childhood care}

\section{Palabras clave}

Atención temprana infantil, calidad, método Visitatie, sello de calidad.

\section{Keywords}

Early childhood care, quality, Visitatie method, quality seal.

\section{Juan Carlos Belda Oriola <beldhern@gmail.com>}

Centro de Atención Pediátrica de Alcoy, Conselleria de Sanidad de la Comunidad Valenciana. España

\section{Antía Rodríguez Suárez} <antia.rguez@gmail.com>

Gabinete de Psicología Arca en Vigo. España

\section{Introducción}

Este artículo recoge el resultado del proyecto estudio elaborado en el seno de la Federación Española de Asociaciones de Profesionales de la Atención Temprana (GAT). Se describe el procedimiento establecido para la obtención del Sello de Calidad Profesional en Atención Temprana, certificación específica para el equipo de profesionales de un Centro de Desarrollo Infantil y Atención Temprana (CDIAT) en su ejercicio profesional. Se realizará una auditoría interna y externa sobre el funcionamiento eficiente y sostenible del equipo, con independencia de la existencia de dispositivos materiales y condiciones laborales mínimas.

Este proyecto pretende ofrecer una herramienta accesible a los profesionales que quieran acreditar la calidad en su quehacer profesional dentro de la atención temprana.

Los evaluadores internos y los externos han de ser profesionales en activo de la atención temprana, con experiencia laboral y formación específica en calidad; cuya interacción pretende tener efectos sinérgicos.

La obtención del sello de calidad avalará que el centro cumple los requisitos mínimos exigidos, además se podrá expedir un certificado individual a los

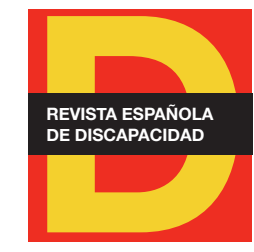

Para citar:

Belda, J. C. y Rodríguez, A. (2020). "Sello de calidad profesional en atención temprana". Revista Española de Discapacidad, 8(2), pp. 231-246.

Doi: <https://doi.org/10.5569/23405104.08.02.12> 
profesionales que han participado en el proceso. La vigencia será de dos años, de forma que se disponga tiempo suficiente para la ejecución de las acciones de mejora previstas y se puedan reevaluar los logros alcanzados.

\section{Fundamentación}

El Libro blanco de la atención temprana (GAT, 2000) delimita claramente los objetivos, definición y ámbitos de intervención, establece un denominador común para los profesionales y para los usuarios, bien sean las familias (EDIS, 2000), las administraciones responsables de la financiación o la gestión de los centros (CDIATs).

En el libro blanco se recogen unos principios básicos como ideario vertebrador de este proceso de cualificación: diálogo, integración y participación, gratuidad, universalidad e igualdad de oportunidades, responsabilidad pública, interdisciplinariedad, alta cualificación profesional, coordinación, descentralización y sectorización.

Tres nociones del libro blanco nos llevan a la interdisciplinariedad:

1. La ampliación del objetivo de atención: a la familia y al entorno.

2. La ampliación de la población diana: a todos los niños con trastornos en el desarrollo o riesgo de padecerlos.

3. La ampliación del momento de intervención: a la prevención en los grupos de riesgo (prevención secundaria), y a la instauración de programas que afectan a toda la población infantil (prevención primaria).

La interdisciplinariedad constituye uno de los principios básicos de la atención temprana, debe estar presente en los tres momentos básicos de la atención temprana: detección, derivación y tratamiento.

En atención temprana (Pérez y Brito, 2004) todo acercamiento e intervención (Alkon, 2007: 366-370) ha de atender a la globalidad del niño, su realidad individual, las relaciones con el resto de la familia, las propias relaciones y de su familia con el entorno. Toda intervención parcial no puede ser considerada atención temprana.

La interdisciplinariedad debe estar presente en todo el "circuito" por el cual pasa el niño o niña y su familia, susceptibles de recibir atención. En la fase de diagnóstico, proporcionar a los profesionales y a las familias la información necesaria acerca de la etiología de los trastornos del desarrollo permitirá establecer conjuntamente un programa personalizado de intervención, la derivación a partir del diagnóstico inicial, la intervención en los CDIATs y la posterior derivación a otros recursos.

La existencia de un programa de atención temprana sectorizado facilita la eficacia del "circuito" atencional, y contemplará la organización y coordinación entre los diversos recursos sanitarios, educativos y de servicios sociales. 
Dentro del CDIAT se posibilitará el trabajo interdisciplinar y se dispondrá de un modelo de gestión que permita alcanzar los objetivos de la calidad total. Situándonos dentro de un programa de atención temprana que este sectorizado, dotado de los recursos adecuados, que coincida con una legislación racional y ajustada a los principios expresados en el Libro blanco de atención temprana.

Tres tipologías de interacción posible entre los profesionales y sus disciplinas dentro equipos de atención temprana:

a. El equipo multidisciplinario, caracterizado por el "intercambio de información". Los miembros del equipo toman las decisiones sobre su respectiva área técnica y realizan partes concretas del programa de intervención con independencia unos de otros. Los informes son específicos de cada profesional. El intercambio de la información pretende presentar los objetivos de cada disciplina y no coordinarse con las demás. La interacción es mínima y no se requiere el consenso del equipo.

b. El equipo interdisciplinario, caracterizado por "compartir y coordinar la información". Cada profesional desarrolla las tareas que le son específicas y se relaciona para coordinar los esfuerzos. Existe un procedimiento formalizado para compartir la información entre los componentes del equipo. Cada intervención complementa los objetivos de las otras disciplinas. Se establecen objetivos comunes, un programa de intervención compartido y se genera la posibilidad de influirse mutuamente. Suele existir un coordinador que facilita el intercambio de la información.

c. El equipo transdisciplinario, caracterizado por "compartir y coordinar tanto la información como los roles". Los distintos profesionales adquieren conocimientos de las otras disciplinas y los incorporan a su propia práctica. Los datos obtenidos se discuten en equipo, se planifican los objetivos y las actuaciones; y se realizan informes integrados. La familia forma parte del equipo en la toma de decisiones. Un profesional y la familia ejecutan el programa individual, con el asesoramiento del resto del equipo.

El equipo transdisciplinar se ajusta más a los objetivos de la atención temprana. Se recomienda esta dinámica como una buena práctica para la intervención ante una serie de colectivos infantiles. Otras intervenciones concretas y puntuales podrán realizarse de forma más individual e independiente del resto del equipo.

Para la configuración definitiva del sello de calidad se debe añadir la "cultura de calidad"; incorporar criterios de calidad en los CDIATs ofrece aportaciones inmediatas, como mejorar y optimizar los procesos de gestión y de intervención (Romero-Galisteo et al., 2015: 71-76), pero al mismo tiempo tiene un valor estratégico de consolidación de la atención temprana ante otros profesionales y ante la sociedad. Una parte importante de la atención temprana ha sido delegada por las autoridades competentes a los distintos agentes de los servicios sociales: como las asociaciones de padres, mancomunidades, etc.; poder justificar la optimización en el uso de los recursos públicos debe constituir un derecho y puede que se convierta en una necesidad.

Tres objetivos básicos en el desarrollo del contenido de la calidad: $1^{\circ}$ ) La inspección de calidad sobre productos finales; $2^{\circ}$ ) El aseguramiento de la calidad a través del control de procesos para garantizar la eficacia y la eficiencia; y $3^{\circ}$ ) La calidad total dirigida a la mejora continua y con la participación de todos en la empresa. El criterio de calidad asistencial más importante de nuestro sistema es la prestación del servicio en tiempo y forma adecuado, pero también existen otros criterios, como una amplia información o el buen trato de los usuarios, que permitirán obtener un valor añadido a la propia asistencia. 
La calidad total "es una estrategia de gestión de las organizaciones cuyo objetivo es la satisfacción de una manera equilibrada de las necesidades y expectativas de los clientes, los empleados, de los accionistas y de la sociedad en general".

Los principios de la gestión de la calidad total son: orientación hacia los resultados, orientación al cliente, liderazgo y constancia en los objetivos, gestión por procesos y hechos, desarrollo e implicación de las personas, aprendizaje, innovación y mejora continua, desarrollo de alianzas y responsabilidad social.

En este presente proyecto se ha elaborado un sistema de evaluación ecléctico para la adjudicación del Sello de Calidad Profesional que incluye la filosofía del modelo EFQM, la metodología del método Visitatie e instrumentos como una adaptación de la Guía de estándares de calidad en atención temprana, la estructura de la ODAT y los análisis DAFO y CAME.

Se requiere de una organización asociativa profesional que asuma la responsabilidad de la organización y verificación del uso correcto del protocolo. Responsabilidad que puede ser compartida con algún departamento de la administración pública competente en el área de la atención temprana.

\section{Metodología}

La adjudicación del sello de calidad se conseguirá mediante un sistema de evaluación basado en el método Visitatie (Perneger, 2006: 397-402) y con la cumplimentación de la 'Batería de indicadores de calidad', el ajuste al procedimiento de trabajo que contiene la ODAT y los análisis DAFO y CAME.

El método Visitatie de evaluación externa por "pares" tiene como característica propia y diferencial que es iniciado y coordinado por los propios profesionales, se centra en la mejora de la asistencia, el intercambio de ideas y se enfatiza más en el aprendizaje que en el control. El objetivo es mejorar la calidad con la concreción de un plan de mejora.

Cuatro objetivos a alcanzar con respecto a las características de un proceso de certificación:

1. Un modelo adecuado a las pequeñas empresas. Los modelos oficiales están diseñados para grandes empresas que disponen de un departamento de personal y de calidad. En un CDIAT no se puede conseguir esa disponibilidad, por falta de medios humanos y de tiempo.

2. Un modelo que considere la estructura horizontal del trabajo transdisciplinar (GAT, 2005) de la atención temprana.

3. Un modelo que entienda la certificación como un medio para la mejora continua, mediante la participación y el crecimiento de todo el equipo de profesionales.

4. Un modelo que recoja estándares significativos para la atención temprana, basado en la relación personal entre los profesionales y los usuarios. 
Estudio comparativo con otros sistemas de certificación:

1. Guía de estándares de calidad en atención temprana. IMSERSO (Ponte et al., 2004).

2. Estándares en centros de atención infantil temprana. JUNTA DE ANDALUCÍA (ACSA, 2012).

3. Borrador proyecto certificación Catalunya. ACAP.

4. ISO.

5. Sello de calidad GAT.

Tabla 1. Sistemas de certificación
\begin{tabular}{|l|c|c|c|}
\hline Certificaciones & Niveles/Criterios/Bloques & $\begin{array}{c}\text { Estándares/Subcriterios/ } \\
\text { Descriptores }\end{array}$ & $\begin{array}{c}\text { Indicadores/Elementos } \\
\text { de medida }\end{array}$ \\
\hline GCAT & 5 & 37 & 144 \\
\hline E-CAIT & 5 & 11 & 83 \\
\hline EFQM-ACAP & 9 & 32 & 294 \\
\hline ISO & & & 140 \\
\hline Sello GAT & 4 & 43 & \\
\hline
\end{tabular}

Fuente: elaboración propia.

El ajuste al procedimiento ODAT (Belda, 2006a) se basa en el proceso de intervención en atención temprana: detección, diagnóstico, tratamiento y evaluación. Una vez detectado el caso se identifican los factores de riesgo y se establecen los trastornos presentes (niveles I y II de la ODAT). El equipo del CDIAT elabora el 'Plan individual de atención temprana'. El Nivel III de la ODAT constituye una hoja de ruta incluyendo las tres fases relevantes que acompañan al proceso diagnóstico: identificar recursos propios, identificar necesidades y diseñar los apoyos que destinados a atender esas necesidades. Dichas fases siempre estarán referidas al niño o niña, la familia (McWilliams, 2009: 1650-1659) y el entorno.

En atención temprana todo el proceso de intervención es susceptible de variaciones según se va avanzando. El propio diagnóstico o diagnósticos iniciales pueden remitir, evolucionar o transformarse, de igual manera que puede variar la situación personal del menor, de la familia y de su entorno. Los apoyos (Shalock, 2004), establecidos mediante las correspondientes UMAT (Unidad de Medida en Atención Temprana) deberán ser variables y flexibles, adaptándose continuamente a las necesidades cambiantes. Variabilidad que se va a establecer en función de las necesidades actuales (Schalok y Verdugo, 2006), en sus distintas fases o realidades.

La eficacia en la atención temprana debe estar fundamentada en la atención específica y adecuada a la diversidad (UNICEF, 2015). Esto exige de intervenciones personalizadas a cada caso y variables a lo largo del tiempo, requisito necesario para conseguir la eficacia en la atención temprana. Constituye un reto, tanto para familias y profesionales, como para la administración por tener que atender a la complejidad sin caer en la tentación de homogeneizar la intervención y evitar el establecimiento de criterios reduccionistas (como la financiación asociada únicamente al número de casos atendidos o al número de sesiones de tratamiento 
ambulatorio); deberán establecer sistemas de financiación y de supervisión sensibles a dicha variabilidad, que además sean sencillos y claros en su implantación. Los CDIAT deberán ser flexibles, adaptarse a las necesidades cambiantes, presentar los resultados de sus intervenciones con transparencia, y establecer protocolos con evidencia científica, siempre y cuando estos protocolos sean capaces de atender este aspecto de la atención temprana y su consecuencia lógica referida a la flexibilidad en el volumen y las características de las intervenciones.

El análisis DAFO establece el estudio de la situación competitiva de una empresa en su mercado (situación externa) y de las características propias (situación interna) de la misma. La situación interna se compone de dos grupos de factores: las fortalezas y las debilidades, la situación externa se compone de otros dos grupos de factores: las oportunidades y las amenazas. Es la herramienta estratégica por excelencia más utilizada para conocer la situación real en que se encuentra la organización.

El análisis consta de cuatro pasos: análisis externo, análisis interno, confección de la matriz DAFO y determinación de la estrategia.

\section{- El análisis externo}

El análisis externo permite fijar las oportunidades y amenazas que el contexto puede presentarle a una organización. El proceso es el siguiente:

a. Establecer los principales hechos o eventos del ambiente que tiene o podrían tener alguna relación con la organización. Pueden ser: de carácter político, legal, social y tecnológico.

b. Determinar qué factores podrían tener influencia sobre la organización en términos de facilitar o restringir el logro de objetivos. Hay circunstancias o hechos presentes en el ambiente que representan una oportunidad que la organización podría aprovechar, para desarrollarse o para resolver un problema. Puede haber situaciones que representen amenazas para la organización y agraven sus problemas. Aplicado a la atención temprana las amenazas constituyen los factores de riesgo y las oportunidades los apoyos.

\section{- Oportunidades}

Las oportunidades son aquellas situaciones externas, positivas, que se generan en el entorno y que pueden ser aprovechadas. Algunas de las preguntas a realizar, que contribuyen en el desarrollo son: ¿A qué oportunidades se enfrenta la empresa? ¿De qué tendencias del mercado se tiene información? ¿Existe una coyuntura en la economía del país? ¿Qué cambios de tecnología observamos? ¿Qué cambios hay en la normatividad legal? ¿Qué cambios encontramos en los patrones sociales y de estilos de vida?

Las oportunidades en atención temprana son factores externos que pueden suponer un apoyo que permita el mejor desarrollo posible (Nivel III de la ODAT sobre los apoyos implementados desde el CDIAT).

\section{- Amenazas}

Las amenazas son situaciones externas, negativas, que pueden atentar contra el proyecto, siendo necesario diseñar una estrategia adecuada para minimizarlas. Algunas de las preguntas a realizar, que contribuyen en el desarrollo son: ¿A qué obstáculos se enfrenta la empresa? ¿Qué están haciendo los competidores? ¿Existen problemas de capital? ¿Pueden impedir la actividad de la empresa? 
Las amenazas en atención temprana son aquellos factores propios o del entorno que pueden impedir o alterar el desarrollo normal del niño o la niña (Nivel I de la ODAT sobre los factores de riesgo).

\section{- El análisis interno}

El análisis interno permite fijar las fortalezas y debilidades de la organización, conocer la cantidad y calidad de los recursos y procesos. Para realizar el análisis interno se identifica qué atributos le permiten generar una ventaja competitiva sobre el resto de sus competidores. Aplicado a la atención temprana las fortalezas constituyen los recursos, y las debilidades vienen clasificadas y definidas en los trastornos.

\section{- Fortalezas}

Las fortalezas son elementos internos y positivos que diferencian al programa o proyecto de otros. Algunas de las preguntas a realizar, que contribuyen en el desarrollo son: ¿Qué ventajas tiene la empresa? ¿Qué hace la empresa mejor que cualquier otra? ¿A qué recursos de bajo coste o de manera única se tiene acceso? ¿Qué percibe la gente como una fortaleza?

Las fortalezas en atención temprana son las capacidades, recursos propios, niveles de maduración alcanzados, constituyen ventajas que sirven para potenciarse junto con las oportunidades: Nivel III de la ODAT sobre los recursos propios del niño o la niña, su familia y su entorno (Belda, 2006b).

\section{- Debilidades}

Las debilidades se refieren a elementos, recursos, habilidades y actitudes que la empresa ya tiene, que constituyen barreras para lograr la buena marcha de la organización. Las debilidades son problemas internos que pueden y deben eliminarse. Algunas de las preguntas a realizar, que contribuyen en el desarrollo son: ¿Qué se puede mejorar? ¿Qué se debería evitar? ¿Qué percibe la gente del mercado como una debilidad? ¿Qué factores reducen las ventas o el éxito del proyecto?

Las debilidades en atención temprana son aspectos que limitan o reducen la capacidad de desarrollo efectivo. Constituyen un impedimento para la calidad de vida, deben ser controladas e intervenidas para paliar sus efectos (Nivel II de la ODAT que hace referencia a los trastornos).

Las potencialidades (combinación de fortalezas y oportunidades) señalan las líneas de acción para la organización. Las limitaciones (combinación de debilidades y amenazas) colocan una seria advertencia.

Los riesgos (combinación de fortalezas y amenazas) y los desafíos (combinación de debilidades y oportunidades) exigirán una cuidadosa consideración a la hora de marcar el rumbo hacia el futuro deseable. Al comparar el análisis DAFO con la estructura de la ODAT (GAT, 2012) se constata la total coincidencia entre ambos instrumentos: uno de análisis de calidad, el otro de procedimiento de intervención mediante el análisis de los puntos fuertes y débiles, internos y externos del niño o la niña. De forma gráfica tenemos: 
Figura 1. ODAT, proceso de atención temprana y análisis DAFO
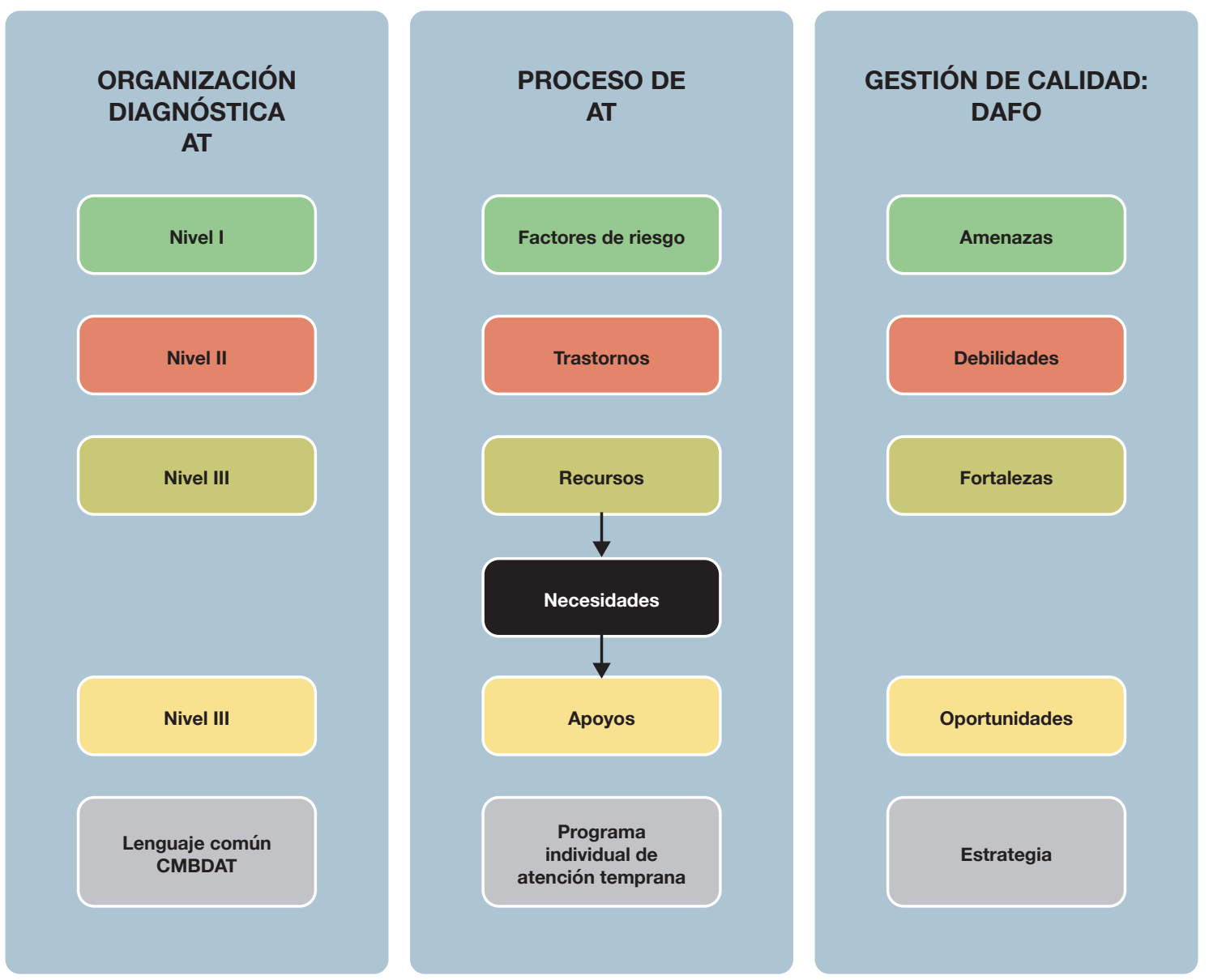

Fuente: elaboración propia.

\section{- La confección de la matriz DAFO}

\section{Tabla 2. Matriz DAFO}

\begin{tabular}{|l|l|}
\hline Análisis interno & Análisis externo \\
\hline Debilidades & Amenazas \\
\hline- & - \\
\hline- & - \\
\hline- & - \\
\hline Fortalezas & Oportunidades \\
\hline- & - \\
\hline- & - \\
\hline- & - \\
\hline
\end{tabular}

Fuente: elaboración propia. 


\section{- Determinar la estrategia a través del análisis CAME}

Tras recopilar cada uno de los factores se desarrolla una estrategia para consolidar las fortalezas, minimizar las debilidades, aprovechar las ventajas y oportunidades del entorno y reducir las consecuencias de las amenazas externas al CDIAT o equipo de profesionales. En esto consiste el análisis CAME (Corregir, Afrontar, Mantener, Explorar).

\section{Tabla 3. Matriz CAME}

\begin{tabular}{|c|c|}
\hline CORREGIR DEBILIDADES & MANTENER FORTALEZAS \\
\hline $\begin{array}{c}\text { Estrategia D/O. Vencer debilidades aprovechando } \\
\text { Oportunidades }\end{array}$ & Estrategia F/A. Usar fortalezas para evitar amenazas \\
\hline- & - \\
\hline- & - \\
\hline AFRONTAR LAS AMENAZAS & EXPLORAR LAS OPORTUNIDADES \\
\hline amenazas & Estrategia F/O. Uso de fortalezas para aprovechar \\
oportunidades
\end{tabular}

Fuente: elaboración propia.

\section{Procedimiento}

\subsection{Proceso}

Se ha estructurado en tres fases:

- La primera fase incluirá la solicitud (A) y el documento de autorización del responsable (B). La segunda fase incluirá evaluación interna (C) y evaluación externa (D). La tercera fase constituirá la aprobación, expedición y registro (E) del sello de calidad.

A. La solicitud la podrán realizar profesionales que trabajen en un CDIAT y que pertenezcan a una organización asociativa de profesionales de atención temprana. Cuando en un CDIAT sean menos de seis trabajadores, la solicitarán un mínimo de dos, cuando haya seis o más profesionales, la solicitarán un mínimo de tres (formulario A-1).

B. Documento de autorización del titular del CDIAT para la realización del protocolo. Se requerirá la firma del titular del CDIAT o persona en la que delegue (dirección técnica) para que conste la autorización explícita a la evaluación interna y externa, en plazo de tiempo establecido (formulario A-2). 
C. Evaluación interna de calidad realizada por la comisión de calidad creada a tal efecto y cuyos miembros han recibido la formación pertinente y específica de calidad en atención temprana. Realizada por tres profesionales, en coordinación con el resto del equipo, que tendrán que rellenar dos documentos en función de las características de su CDIAT: la batería de indicadores y la matriz DAFO-CAME (formularios A-3 y A-4).

\begin{tabular}{|c|c|c|c|}
\hline Comunidad & CDIAT & PIAT & Profesionales \\
\hline Detección & Proceso interdisciplinario & Valoración inicial & Funciones director CDIAT \\
\hline Protocolos cribado & Equipo multiprofesional & Recursos propios & Coordinador caso \\
\hline Derivación & Proceso participativo & Recursos institucionales & Funciones en el CDIAT \\
\hline Solicitud & Coordinador caso & Necesidades & $\begin{array}{c}\text { Funciones con el responsable } \\
\text { del caso }\end{array}$ \\
\hline Sectorización & Responsable caso & Apoyos personales & Funciones con el niño \\
\hline \multirow[t]{12}{*}{ Evaluación externa } & Gestión y administración & Apoyos materiales & Funciones con la familia \\
\hline & Derechos y deberes & $\begin{array}{c}\text { Programa individual atención } \\
\text { temprana }\end{array}$ & Funciones con el entorno \\
\hline & Coordinación & Programa intervención directa & Responsable caso \\
\hline & Satisfacción & Apoyo escolarización & Funciones con el coordinador \\
\hline & Investigación & Participación social & Funciones con el niño \\
\hline & Formación & Revisión periódica & Funciones con la familia \\
\hline & Cartera de servicios & & Funciones con el entorno \\
\hline & Prevención y detección & & \\
\hline & Evaluación & & \\
\hline & Proyecto de actividades & & \\
\hline & Memoria anual & & \\
\hline & $\begin{array}{l}\text { Seguridad y prevención de } \\
\text { accidentes }\end{array}$ & & \\
\hline
\end{tabular}

Fuente: elaboración propia.

Evaluación externa por parte de dos expertos profesionales pertenecientes a una organización asociativa de profesionales y con la formación específica en calidad. Realizada mediante una visita al CDIAT, incluyendo entrevistas al responsable del centro, a otros profesionales, a usuarios, tanto familiares como a usuarios en sesión, si resultara de interés visitas al entorno (escuelas infantiles, hospital, etc.) y el estudio y análisis de los documentos aportados. Valoración de la documentación propia y de la aportada por la comisión interna. La redacción del análisis CAME deberá consensuarse conjuntamente por los componentes de las dos comisiones (formulario A-5). 
Tabla 5. Respuestas batería de indicadores

\begin{tabular}{|c|c|c|c|c|c|c|c|c|c|c|c|}
\hline $\mathbf{N}^{\circ}$ & $\mathbf{N}$ & A/V & $s$ & $\mathbf{N}^{\circ}$ & $\mathbf{N}$ & A/V & $\mathrm{s}$ & $\mathbf{N}^{\circ}$ & $\mathbf{N}$ & $\mathbf{A} / \mathbf{V}$ & $\mathrm{s}$ \\
\hline 1.01 & & & & 2.34 & & & & 3.31 & & & \\
\hline 1.02 & & & & 2.35 & & & & 3.32 & & & \\
\hline 1.03 & & & & 2.36 & & & & 3.33 & & & \\
\hline 1.04 & & & & 2.37 & & & & 3.34 & & & \\
\hline 1.05 & & & & 2.38 & & & & 3.35 & & & \\
\hline 1.06 & & & & 2.39 & & & & 3.36 & & & \\
\hline 1.07 & & & & 2.40 & & & & 3.37 & & & \\
\hline 1.08 & & & & 2.41 & & & & 3.38 & & & \\
\hline 1.09 & & & & 2.42 & & & & 3.39 & & & \\
\hline 1.10 & & & & 2.43 & & & & 3.40 & & & \\
\hline 1.11 & & & & 2.44 & & & & 3.41 & & & \\
\hline 1.12 & & & & 2.45 & & & & 3.42 & & & \\
\hline 1.13 & & & & 2.46 & & & & 3.43 & & & \\
\hline 1.14 & & & & 2.47 & & & & 3.44 & & & \\
\hline 2.01 & & & & 2.48 & & & & 4.01 & & & \\
\hline 2.02 & & & & 2.49 & & & & 4.02 & & & \\
\hline 2.03 & & & & 2.50 & & & & 4.03 & & & \\
\hline 2.04 & & & & 3.01 & & & & 4.04 & & & \\
\hline 2.05 & & & & 3.02 & & & & 4.05 & & & \\
\hline 2.06 & & & & 3.03 & & & & 4.06 & & & \\
\hline 2.07 & & & & 3.04 & & & & 4.07 & & & \\
\hline 2.08 & & & & 3.05 & & & & 4.08 & & & \\
\hline 2.09 & & & & 3.06 & & & & 4.09 & & & \\
\hline 2.10 & & & & 3.07 & & & & 4.10 & & & \\
\hline 2.11 & & & & 3.08 & & & & 4.11 & & & \\
\hline 2.12 & & & & 3.09 & & & & 4.12 & & & \\
\hline 2.13 & & & & 3.10 & & & & 4.13 & & & \\
\hline 2.14 & & & & 3.11 & & & & 4.14 & & & \\
\hline 2.15 & & & & 3.12 & & & & 4.15 & & & \\
\hline 2.16 & & & & 3.13 & & & & 4.16 & & & \\
\hline 2.17 & & & & 3.14 & & & & 4.17 & & & \\
\hline 2.18 & & & & 3.15 & & & & 4.18 & & & \\
\hline 2.19 & & & & 3.16 & & & & 4.19 & & & \\
\hline 2.20 & & & & 3.17 & & & & 4.20 & & & \\
\hline 2.21 & & & & 3.18 & & & & 4.21 & & & \\
\hline 2.22 & & & & 3.19 & & & & 4.22 & & & \\
\hline 2.23 & & & & 3.20 & & & & 4.23 & & & \\
\hline 2.24 & & & & 3.21 & & & & 4.24 & & & \\
\hline 2.25 & & & & 3.22 & & & & 4.25 & & & \\
\hline
\end{tabular}




\begin{tabular}{|c|l|l|l|l|l|l|l|l|l|l|l|}
\hline $\mathbf{N}^{\circ}$ & $\mathbf{N}$ & $\mathbf{A} / \mathbf{V}$ & $\mathbf{S}$ & $\mathbf{N}^{\mathbf{0}}$ & $\mathbf{N}$ & $\mathbf{A} / \mathbf{V}$ & $\mathbf{S}$ & $\mathbf{N}^{\circ}$ & $\mathbf{N}$ & $\mathbf{A} / \mathbf{V}$ & $\mathbf{S}$ \\
\hline 2.26 & & & & 3.23 & & & & 4.26 & & & \\
\hline 2.27 & & & & 3.24 & & & & 4.27 & & & \\
\hline 2.28 & & & & 3.25 & & & & 4.28 & & & \\
\hline 2.29 & & & & 3.26 & & & & 4.29 & & & \\
\hline 2.30 & & & & 3.27 & & & & 4.30 & & & \\
\hline 2.31 & & & & 3.28 & & & & 4.31 & & & \\
\hline 2.32 & & & & 3.29 & & & & 4.32 & & & \\
\hline 2.33 & & & & 3.30 & & & & Total & & & \\
\hline
\end{tabular}

Fuente: elaboración propia.

Una vez evaluados todos los documentos y los procedimientos, los evaluadores externos darán el visto bueno a la concesión del sello de calidad, lo que notificarán a la organización asociativa de profesionales para su aprobación, registro y expedición al CDIAT y a los profesionales socios (formulario A-6 y A-7). En caso de que no se reunieran los requisitos mínimos se notificaría a la comisión interna de calidad.

\subsection{Interpretación resultados batería de indicadores}

Equivalencia en puntuaciones: "Nunca" = 0 puntos, "A veces" = 1 punto, "Siempre" = 2 puntos. Si disponemos de 140 indicadores la puntuación podrá oscilar entre 0 y 280 puntos. Una vez revisado el cuestionario de la batería de indicadores se obtendrá una puntuación que se registrará en el formulario A-6 de resultados de la evaluación.

El mínimo de puntuación establecido está constituido por los siguientes indicadores: bloque $2(01,02,04$, $05,06,07,08,10,15,20,21,22,23,25,30,32,34,35,42,45,47,49)$ y bloque $3(01,02,03,10,11,12,13$, $14,16,18,23,28,30,33,35,38,44)$

Tabla 6. Indicadores mínimos

Tabla 6. Indicadores mínimos
\begin{tabular}{|c|c|c|c|c|c|c|c|c|c|c|c|}
\hline $\mathbf{N}^{\circ}$ & $\mathbf{N}$ & $\mathbf{A} / \mathbf{V}$ & $\mathbf{S}$ & $\mathbf{N}^{\circ}$ & $\mathbf{N}$ & $\mathbf{A} / \mathbf{V}$ & $\mathbf{S}$ & $\mathbf{N}^{\circ}$ & $\mathbf{N}$ & $\mathbf{A} / \mathbf{V}$ & $\mathbf{S}$ \\
\hline 1.01 & & & & 2.34 & & & & 3.31 & & & \\
\hline 1.02 & & & & 2.35 & & & & 3.32 & & & \\
\hline 1.03 & & & & 2.36 & & & & 3.33 & & & \\
\hline 1.04 & & & & 2.37 & & & & 3.34 & & & \\
\hline 1.05 & & & & 2.38 & & & & 3.35 & & & \\
\hline 1.06 & & & & 2.39 & & & & 3.36 & & & \\
\hline 1.07 & & & & 2.40 & & & & 3.37 & & & \\
\hline 1.08 & & & & 2.41 & & & & 3.38 & & & \\
\hline 1.09 & & & & 2.42 & & & & 3.39 & & & \\
\hline 1.10 & & & & 2.43 & & & & 3.40 & & & \\
\hline 1.11 & & & & 2.44 & & & & 3.41 & & & \\
\hline
\end{tabular}




\begin{tabular}{|c|c|c|c|c|c|c|c|c|c|c|c|}
\hline $\mathbf{N}^{\circ}$ & $\mathbf{N}$ & A/V & $\mathrm{s}$ & $\mathbf{N}^{\circ}$ & $\mathbf{N}$ & $\mathrm{A} / \mathrm{V}$ & $\mathrm{s}$ & $\mathbf{N}^{\circ}$ & $\mathbf{N}$ & $\mathrm{A} / \mathrm{V}$ & s \\
\hline 1.12 & & & & 2.45 & & & & 3.42 & & & \\
\hline 1.13 & & & & 2.46 & & & & 3.43 & & & \\
\hline 1.14 & & & & 2.47 & & & & 3.44 & & & \\
\hline 2.01 & & & & 2.48 & & & & 4.01 & & & \\
\hline 2.02 & & & & 2.49 & & & & 4.02 & & & \\
\hline 2.03 & & & & 2.50 & & & & 4.03 & & & \\
\hline 2.04 & & & & 3.01 & & & & 4.04 & & & \\
\hline 2.05 & & & & 3.02 & & & & 4.05 & & & \\
\hline 2.06 & & & & 3.03 & & & & 4.06 & & & \\
\hline 2.07 & & & & 3.04 & & & & 4.07 & & & \\
\hline 2.08 & & & & 3.05 & & & & 4.08 & & & \\
\hline 2.09 & & & & 3.06 & & & & 4.09 & & & \\
\hline 2.10 & & & & 3.07 & & & & 4.10 & & & \\
\hline 2.11 & & & & 3.08 & & & & 4.11 & & & \\
\hline 2.12 & & & & 3.09 & & & & 4.12 & & & \\
\hline 2.13 & & & & 3.10 & & & & 4.13 & & & \\
\hline 2.14 & & & & 3.11 & & & & 4.14 & & & \\
\hline 2.15 & & & & 3.12 & & & & 4.15 & & & \\
\hline 2.16 & & & & 3.13 & & & & 4.16 & & & \\
\hline 2.17 & & & & 3.14 & & & & 4.17 & & & \\
\hline 2.18 & & & & 3.15 & & & & 4.18 & & & \\
\hline 2.19 & & & & 3.16 & & & & 4.19 & & & \\
\hline 2.20 & & & & 3.17 & & & & 4.20 & & & \\
\hline 2.21 & & & & 3.18 & & & & 4.21 & & & \\
\hline 2.22 & & & & 3.19 & & & & 4.22 & & & \\
\hline 2.23 & & & & 3.20 & & & & 4.23 & & & \\
\hline 2.24 & & & & 3.21 & & & & 4.24 & & & \\
\hline 2.25 & & & & 3.22 & & & & 4.25 & & & \\
\hline 2.26 & & & & 3.23 & & & & 4.26 & & & \\
\hline 2.27 & & & & 3.24 & & & & 4.27 & & & \\
\hline 2.28 & & & & 3.25 & & & & 4.28 & & & \\
\hline 2.29 & & & & 3.26 & & & & 4.29 & & & \\
\hline 2.30 & & & & 3.27 & & & & 4.30 & & & \\
\hline 2.31 & & & & 3.28 & & & & 4.31 & & & \\
\hline 2.32 & & & & 3.29 & & & & 4.32 & & & \\
\hline 2.33 & & & & 3.30 & & & & Total & & & \\
\hline
\end{tabular}

Fuente: elaboración propia.

En total 23 ítems del bloque 2 más 17 ítems del bloque 3, es decir, 40 indicadores con una puntuación total máxima de 80 puntos. 


\subsection{Pruebas piloto}

Tres etapas:

1. Planificación del protocolo: selección, cribado e incorporación de indicadores tomando como base otras guías o tablas de estándares o indicadores, específicos de atención temprana.

2. Primera prueba piloto: cuatro CDIATs (CDIAT Arca de Vigo, CDIAT de Santiago de Compostela, CDIAT Marina Alta de Denia y Centro de Atención Pediátrica de Alcoy). Con una doble tarea: aplicar el protocolo interviniendo como evaluadores internos y como evaluadores externos con el centro en que se emparejaron; evaluar el protocolo en su totalidad.

Terminado el proceso se realizó un profundo cambio tanto en los bloques como en los indicadores.

3. Segunda prueba piloto: participaron 24 CDIATs:

Comunidad Valenciana: CAT L'Horta Nord (Alboraya)

ATTEM (Valencia)

CDIAT Luis Suñer Sanchis (Alzira)

CAT L'Horta Nord (Massamagrell)

CDIAT Enrique Riquelme (Paterna)

CUDAP (Valencia)

ASPAS CASTELLÓN-HELIX (Castellón de la Plana)

CDIAT Marina Alta (Denia)

Centro Municipal de Atención Temprana (Orihuela)

Centro de Atención Pediatrica (Alcoy)

Murcia: $\quad$ Centro Municipal de Desarrollo Infantil y Atención Temprana (Cieza)

CDIAT Fina Navarro López (Lorca)

CDIAT (Águilas)

Centro de Atención Temprana (Fortuna)

CDIAT ADAMUR (San Ginés)

CDIAT ASPAJUNIDE (Jumilla)

CDIAT Fundación ASÍNTER (Murcia)

CDIAT ASPANPAL (Murcia)

CDIAT ASTRADE (Ribera del Molina)

Asociación ATEMYTEA (Murcia)

Galicia:
ADIAF (San Xoan de Tabagón-O Rosal)
Gabinete de Psicologia ARCA (Vigo)
CONVERSA (Vigo)
CDI AGARIMOS (Santiago de Compostela)

Los centros tenían que cumplimentar el protocolo y evaluarlo en su totalidad. Al terminar el proceso se incorporaron mejoras puntuales en los indicadores, para conformar la tabla definitiva que ahora se propone y ofrece en este artículo. También se consensuaron valoraciones y directrices que incluiremos en el apartado de conclusiones. 


\section{Conclusiones}

Con respecto al "protocolo sello" en su conjunto se llegó a las siguientes conclusiones:

- La necesidad de formar a los auditores internos/externos desde una consultoría constituida por la asociacion/federación de profesionales.

- La necesidad de tener a disposición esta consultoría a lo largo del proceso para asesoramiento.

- Establecer los plazos, la logística y las tareas administrativas necesarias, de forma que el proceso completo del sello de calidad pueda estar finalizado en tres meses.

- Mejora y formación en el uso de la documentación informatizada.

- Necesidad de establecer un lenguaje común en cuanto al contenido de los términos utilizados en atención temprana.

- Se valora muy positivamente este procedimiento porque aborda los aspectos profesionales frente a otros sistemas de certificación que son administrativos.

- Permite la reflexión interna y el crecimiento profesional del equipo de atención temprana.

- El proceso DAFO/CAME requiere una formación más intensa.

Tres ideas esenciales, la primera, la consideración generalizada de que el "protocolo sello" recoge la importancia del tipo de relaciones que se establecen entre los agentes de la atención temprana: profesionales, niños y niñas, familias y otros profesionales (Black et al., 2017: 77-90), las relaciones dentro del equipo, etc. La segunda, valorar la participación en el "protocolo sello" como una formación continua de los profesionales, que han expresado su satisfacción al evaluarse ellos mismos y por la enorme aportación que les hace el actuar como evaluadores de sus iguales y poder contrastar formas distintas, metodologías complementarias de actuación. En tercer lugar, valorar como muy positivo la sencillez y aplicabilidad del protocolo en su conjunto, dentro de la dinámica normal de funcionamiento de un CDIAT, por lo que lo recomendamos a todos los centros, no solo en España, sino al resto de Europa, a los hermanos iberoamericanos y a todos en general.

Este sello nos puede servir como Sello Profesional de Calidad en Atención Temprana complementario a otras certificaciones oficiales que ya ostenten los CDIATs o como material básico para la elaboración de un sistema de certificación entre los profesionales y la administración pública con competencias en atención temprana. 


\section{Referencias bibliográficas}

Agencia de Calidad Sanitaria de Andalucía (2012). Manual de estándares de los centros de atención infantil temprana. Sevilla: Junta de Andalucía.

Alkon, A. et al. (2009). "Child care health consultation improves health and safety polices and practices". Acad. Pediatr., 9(5), pp. 366-370.

Black, M. M. et al. (2017). "Early childhood development coming of age: Science through the life course". The Lancet, 389(10064), pp. 77-90.

Belda, J. C. (2006a). "La ODAT: una herramienta para el desarrollo de la atención temprana". Revista Informació Psicològica, 87, pp. 68-80 Valencia: Colegio Oficial de Psicólogos del País Valenciano.

Belda, J. C. (2006b). "Recursos en atención temprana. Nivel III de la ODAT". Revista de atención temprana, 9(1), pp. 37-51.

EDIS (2000). Necesidades, demandas y situación de las familias con menores (0-6 años) discapacitados. Madrid: IMSERSO.

GAT (2000). Libro blanco de la atención temprana. Madrid: Real Patronato de Prevención y Atención a Personas con Minusvalía.

GAT (2005). Recomendaciones técnicas para el desarrollo de la atención temprana. Madrid: Real Patronato sobre Discapacidad.

GAT (2012). Organización diagnóstica para la atención temprana. Madrid: Real Patronato sobre Discapacidad.

McWilliams, C. et al. (2009). "Best practice guidelines for physical activity at child care". Pediatrics, 124(6), pp. 1650-1659.

Pérez-López, J. y Brito, A. G. (2004). Manual de atención temprana. Madrid: Pirámide.

Perneger, T. (2006). "Efectos del 'visitatie' entre los profesionales sanitarios". Internacional Journal for Quality in Health Care, 18(6), pp. 397-402.

Ponte, J. et al. (2004). Guía de estándares de calidad en atención temprana. Madrid: IMSERSO.

Romero-Galisteo, R. P. et al. (2015). "Percepción de la calidad en centros de atención temprana: resultados de un análisis comparativo". Escritos de Psicología, 8(2), pp. 71-76.

Shalock, R. L. et al. (2004). Retraso mental. Definición, clasificación y sistemas de apoyo. Madrid: Alianza Editorial.

Schalock, R. L. y Verdugo, M. A. (2006). Calidad de vida. Manual para profesionales de la educación, salud y servicios sociales. Madrid: Alianza Editorial.

UNICEF Comité Español (2015). Convención sobre los derechos del niño. Madrid: UNICEF. 